\title{
Motor-Sensory and Visual Behavior After Hemispherectomy in Newborn and Mature Rats
}

\author{
Samuel P. Hicks and Constance J. D'Amato ${ }^{1}$ \\ Department of Pathology, University of Michigan Medical Center, \\ Ann Arbor, Michigan 48104
}

Received July 27, 1970 revision received August 29, 1970

\begin{abstract}
Immature mammals are widely believed to compensate functionally for nervous system alterations better than adults with comparable disorders. Embryos restitute huge losses, but plasticity, remodeling, or use of alternate mechanisms said to underly compensation in injured infants are not understood. Toward understanding these, the effects of ablating or altering parts of the nervous system in infant and mature rats are being studied. In these experiments one lateral half of the forebrain and diencephalon was largely removed at birth or maturity and the consequences to nervous system structure and motor-sensory and visual behavior were observed. Similarities between animals operated on as aduits or infants were loss of tactile placing opposite the ablation, ability to discriminate visual patterns, and gauge variable jumping distances visually. Some subjects performed the visual tasks using the eye opposite the hemispherectomy alone, which was exclusively supplied with uncrossed retinogeniculate fibers. Differences were: loss of tactile placing after operation in infants was delayed until the seventeenth day; stride was impaired in animals operated on as adults but was spared in infant subjects; with appropriate ablations, Fink-Heimer-Nauta stains showed that after hemispherectomy, infants, but not adults, developed a small, uncrossed corticospinal tract. The stride component in locomotion seemed dependent on the corticospinal tract system, and was partially dissociated from the placing reaction essential for locomotion on rough terrain. The possibility was considered that the small remodeled corticospinal tract spared the stride component.
\end{abstract}

\section{Introduction}

The immature mammal is said to be able to compensate for nervous system injuries much better than the mature organism. This is clearly true in early embryonic life, when the mammal, regulative like its simpler cousins, can restitute huge losses and grow up, apparently normal (11). Less is known about responses of the fetus and infant; when compensation occurs

1 This research is supported by USPHS Grant NS03861 and Atomic Energy Commission Contract AT(11-1)1201. We thank Paul K. Brandon, Mankato University, James H. Woods, University of Michigan, and Marion Hope, University of Florida, Gainesville for suggestions for the behavioral experiments. 
after an injury expected to cause a functional deficit, it is loosely attributed to plasticity or available alternate mechanisms. Bilateral representation of some functions in both immature and mature brains is an illustration of alternate or redundant mechanisms, but morphologic plasticity or remodeling, certainly anything like that in the regulative embryo, has not been demonstrated.

The problem is illustrated by infant monkeys deprived of motor cortices who grew up to have normal motor function in contrast to adults who were impaired after similar lesions $(15,26)$. Cats were able to see if they lost their visual cortices in infancy but not if the loss occurred later (6). A man born with virtually no cerebellum who got along as a bricklayer's assistant (3), another man with rudimentary hippocampi and no fornices who was mentally bright and normal (23), and a hemispherectomized boy with scarcely appreciable motor and mental impairment, all developed functions that could not have been predicted.

Functional losses in the presence of brain abnormalities may be overlooked if appropriate tests arc not done. The monkeys injured as infants just mentioned, unlike normals, could not remember under which of two containers they saw food placed if the containers were briefly shut off from their view, even for a few seconds. Animals deprived of their hippocampi in infancy did better on certain tasks when they grew up than did animals injured as adults, but on some other tasks the latter had the advantage (14). The problem is further compounded, that ablation of the same part of the nervous system at two stages of life may have different structural consequences and alter function differently. Cerebellectomy in infant rats resulted in a syndrome which differed in several respects from that which it caused in adults (13). And the effects of malformation on function, just beginning to be studied, may be quite different from loss of a part depending on what is malformed and how $(12,13)$.

To understand the responses of the young to injury better, we have ablated or altered parts of the nervous system in infant rats and studied some of the morphogenetic and developing behavioral consequences. Our interest has been especially to find out what injured organisms can still do - "residual performance"-and it has centered on visual and motor function as one means to this end. When possible, comparable studies in adults have been done. This paper describes rats with hemispherectomy.

\section{Methods}

General Plan. Despite evidence of considerable residual bilateral motor function after hemispherectomy in mature monkeys (29), and still other functions in infant and juvenile man and monkey after partial hemispherectomy $(8,24,28)$ our first hypothesis was that nearly complete hemi- 
spherectomy would severely impair function in infants or adults by hemiparalysis or hemiparesis, but that the infants would show some compensation. The first experiments showed no such incapacity in either; the less severe functional changes that we recognized were therefore observed and compared. Locomotion, placing reactions, and visually guided jumping variable distances to a platform were first studied, beginning before weaning in the infant subjects. A paradox of the animals' visual placing reactions recognized as the experiments progressed led to studying the vision of some animals in more detail. The paradox was that some subjects placed the forefoot opposite the ablation neither tactually nor visually with the eye on that side, seeming to be blind in that eye: yet some animals placed if both the tactile and visual stimuli were combined. Also, because the eye opposite the lesion had only uncrossed retinogeniculate fibers there was an opportunity to observe function of those fibers exclusively when the other eye was closed $(16,20,22)$.

Half the brain rostral to the tentorium was largely or nearly completely removed from 22 newborn (12- to 28 -hr old) and six mature rats $(6 \mathrm{r} / 2$ or 10 weeks old). Behavioral studies were then done, some beginning just after the operations. One or more normal sibs in each litter were controls. The 28 animals operated on and 16 controls were killed at 5-14 months, and the brains and spinal cords, and sometimes eyes, of those operated on and controls for each litter were studied histologically. To find out whether the remaining corticospinal tract in either the infant or the mature rat had the "plasticity" to remodel itself after hemispherectomy by distributing fibers to its usual terminations bilaterally in the medulla and spinal cord, ablations were made in the dorsal frontal cortex of the remaining hemisphere of subjects hemispherectomized either as infants or at maturity. The animals' nervous systems were then studied by a Nauta method modified by Fink and Heimer ( 7$)$. The rat has been shown by several methods to have a completely cross corticospinal tract $(1,2)$. To confirm and explore this further, frontal cortical ablations were performed on mature and newborn rats, and in another mature rat hemispherectomy was done, to observe the normal course of the tract by the Fink-Heimer-Nauta stain.

Animals and Facilities. The rats, black with white feet (Irish), were the $F_{1}$ cross of black-selfed nonagouti males, about 50 generations brothersister mated, and albino females of Wistar origin, similarly inbred, in a closed colony. They were usually kept in groups in clear plastic cages with Sanicel bedding, in air-conditioned rooms, $25 \mathrm{C}$, variable humidity, and fluorescent light on 7:00 AM to 11:00 PM. Food was Purina Mouse Breeder Chow. In two of the rooms insulated from ambient noise behavioral studies were done. Both sexes were $5.5-6 \mathrm{~g}$ at birth. Males were 
about $300-330 \mathrm{~g}$ at 4 months, $350-400 \mathrm{~g}$ at 1 year. Females were about $200-270 \mathrm{~g}$ at these ages. Subjects were randomly both sexes.

Ability to reproduce, which began at about 8 weeks in our rats, is a commonly accepted sign of maturity or adulthood. Arbitrarily, we have also considered the rats operated on at $6 / 2$ weeks of age as mature or adult.

Operations. Brain tissue was aspirated, under a dissecting microscope, through a craniotomy, with ether anesthesia. Aspiration and physiologic saline solution rinses provided hemostasis. There were no infections. Two infants died soon after operation; they were in addition to the 22 that grew up. Infants were returned to the mother and littermates within an hour or two after operation when they soon resumed nursing. Adults were severely incapacitated for a couple of days after operation; they were given appropriate subcutaneous glucose in saline solution. All hemispherectomies were on the right except two in infants. The frontal cortex of the remaining hemisphere was also ablated at the time of hemispherectomy in two infants to see what the combined effect would be.

The term "hemispherectomy" is variously used in biomedical publications (28). In these experiments, the aim of the operation was to remove one lateral half of the brain rostral to the tentorium without crossing the midline or attempting to remove all of the olfactory bulb. The result, except in one case, an infant in which much thalamus remained, was a close approximation of the aim with a variable midline residuum of medial thalamic, hypothalamic, and ventral forebrain structures, and medial remnants of the cortical mantle. The region of the optic chiasm was regularly spared. Except that it was severed in one infant, the optic nerve on the side of the operation was spared. In some cases the sagittal plane of the hemispherectomy passed close to or through the midline in most of its extent except the rostral ventral portion. One of the most nearly complete operations is represented in Fig. 1. Medial mantle remnants, which sometimes included the medial parts of the cortex at the poles. kept some of their immediate, superficial midline fiber connections, but there was no centrum ovale, corpus striatum, or internal capsule. The rostral extreme of the cerebral peduncle was encroached upon, but rarely to the level of the exiting oculomotor nerve. The most rostral part of the superior colliculus was sometimes grazed. More details are given in Results.

Motor-sensory Functions. Infant and adult subjects were examined daily for several weeks after operation, less often later. Motion pictures at 64 frame/sec were made of locomotion on a flat, level board runway, viewed horizontally and from above, and in some instances on horizontal ladders; and of jumping and placing; usually at ages 17, 21. and 25 days and 2 months or older in infant subjects, and in the first week and later after operations in mature animals. The runway was nearly $3 \mathrm{~m}$ long, $15 \mathrm{~cm}$ wide, 

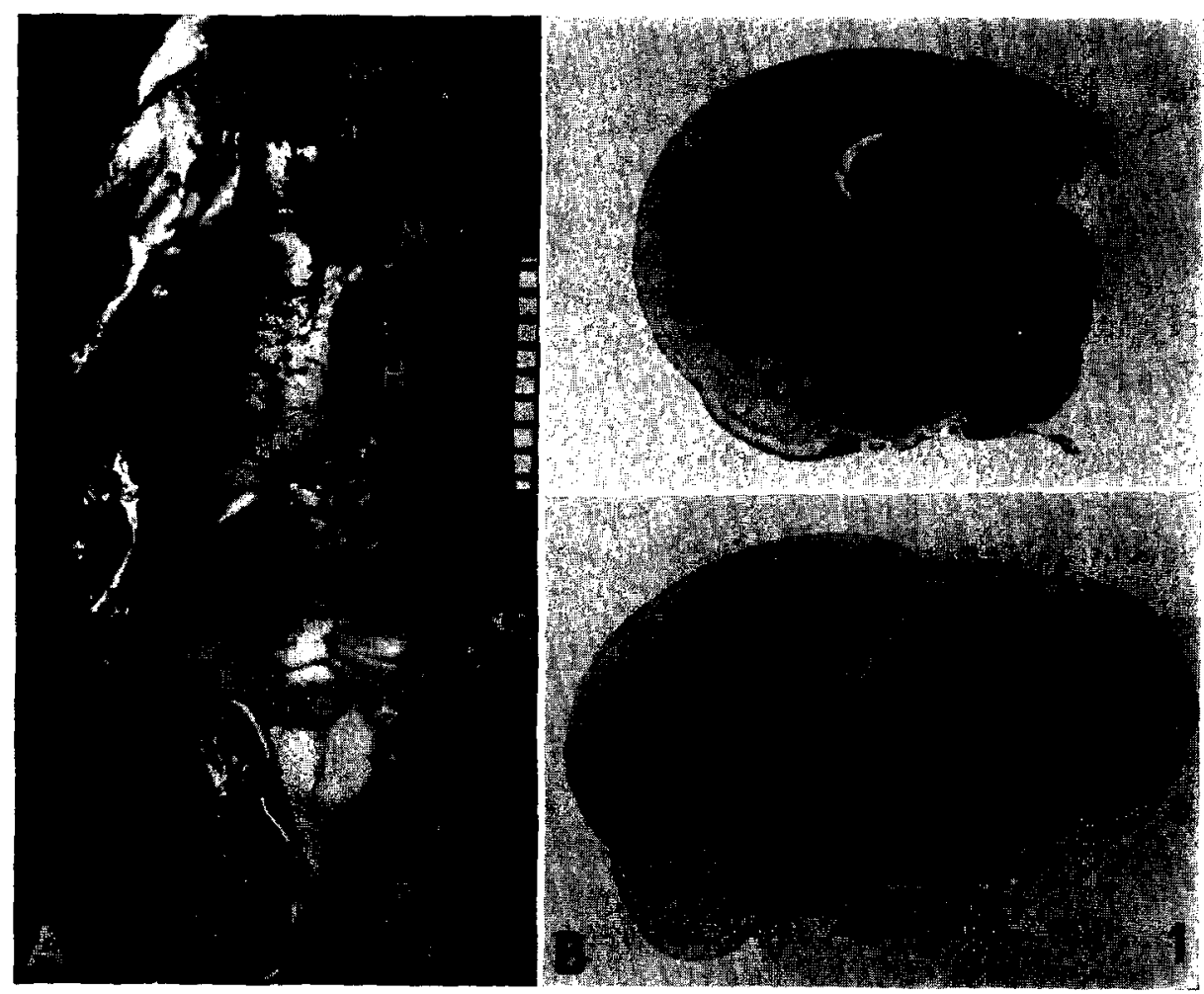

FIG. 1. Brain in situ of an adult rat hemispherectomized as a newborn infant. A. Gross brain showing $\mathrm{H}$, site of hemispherectomy, $\mathrm{M}$, medial mantle remnant, $\mathrm{C}$, inferior colliculus. B. Two frontal sections of the brain, upper at beginning of optic tract, lower at medial geniculate level. In the upper, the ablation passed through the third ventricle ( Luxol fast blue and cresyl violet stain, $\times 5$ ).

curved on a $2.2-\mathrm{m}$ radius for motion pictures, and its front edge was marked at $10-\mathrm{cm}$ intervals. The ladder rungs were square or round, $0.7 \mathrm{~cm}$ in diameter, and spaced $3.5 \mathrm{~cm}$ on centers. The space was an approximate fraction of the usual running strides of animals at the stages photographed. Hopping and stepping reactions, forward and lateral tactile placing with the forefeet, locomotion on a level surface or runway were tested from shortly after operations; standing and visual placing in the young subjects were observed from early in the third week. Coordination of turning and grooming were observed. Righting, response to pinprick and touch at a few points over the body were tested shortly after operation and at later intervals. Controls were tested as the hemispherectomized animals.

In lateral tactile placing the animal lifted its forefoot and placed it in a lateral direction on a horizontal ledge in response to touching the lateral side of the foot to the vertical side of the ledge. It was a precisely executed response from about 8 days of age. It was elicited during the first week, 
but often confounded with spontaneous movements. A similar tactile placing reflex could normally be elicited in the forward direction, but because the lateral response was abolished by hemispherectomy while the forward response was variably affected, we made the arbitrary distinction. Tactile placing by the hind feet was normally usually capricious but sometimes used. The placing was tested, except as noted, with the animals' eyes covered with a mask or the experimenter's fingers, and placing in response to touching vibrissae was avoided.

Visually Guided Jumping. All rats were trained to jump varying distances from one platform to another. Infant subjects and their normal sibs hegan about 15-17 days of age, first stepping across a centimeter gap and increasing the distance by small increments. By 3 weeks they were jumping $20 \mathrm{~cm}$, and $25-30 \mathrm{~cm}$ by 4 weeks, which was between two and three times their body lengths. Mature subjects were begun the same way several days after operation because they fell easily at first. Jumping, in normal light, was begun on large platforns $14 \mathrm{~cm}$ deep, $20 \mathrm{~cm}$ wide, and $24 \mathrm{~cm}$ high, padded with firm sponge rubber traction tops. As $20-\mathrm{cm}$ jumps werc attained, a round jumping platform $13 \mathrm{~cm}$ in diameter was substituted with a landing platform $7 \mathrm{~cm}$ wide and $10 \mathrm{~cm}$ long, both the same height and padded as before. Foam cushions were extensively used around the platforms and on the front edges of the landing platform to prevent possible injury or aversive effects. The distance between the platforms was varied randomly mostly by moving the jumping platform along a line parallel to the wall on the animal's left when the animal was in jumping position, but the positions of both platforms were sometimes varied laterally too (see Controls for Nonvisual Cues, later).

Reinforcement for jumping in young animals until they were 2 or 3 months old and sometimes longer was the junuping, being handled and sparring with the experimenter's fingers, and exploration of the platforms and other places beyond the landing platform. (Pushing against and knocking down the cards bearing patterns in the Lashley visual discrimination apparatus mentioned later was similarly reinforcing to juvenile animals.) Some adult animals, operated on either as infants or adults, were food deprived to $10-15 \%$ free-feeding weight and observed as they jumped varying distances from $15-40 \mathrm{~cm}$ for food reinforcement. This was done on a schedule of daily sessions of about 10 trials over a period of 3 weeks, the purpose to determine whether the hemispherectomized rats differed from normals in gauging distances and precision of jumping. Though the procedure often increased the rapidity of jumping, it gave no more information than less formal and frequent testing.

Some animals were also tested jumping to a landing platform similar to the one mentioned, but with a built-in tiny lamp, controlled by a rheostat, that illuminated the platform surface. The surface was white translucent 
plastic with narrow longitudinal rubber traction strips. Jumping was done in a dark room, where the platform surface appeared to the experimenter as a faintly glowing striped rectangle in black ambience when the lamp was set at dimmest. A Luna-six (P. Gossen \& Co.) photographic light meter, set for incident light, held $2 \mathrm{~cm}$ directly above the platform indicated approximately $0.02 \mathrm{ft}-\mathrm{c}$ at this setting. In a single session of about $20 \mathrm{~min}$, the animals were introduced to this platform a few centimeters away from their jumping platform, with the light set at intermediate brightness. The distance was increased in about 8-10 steps to $30 \mathrm{~cm}$, and the light dimmed progressively.

Some animals were also trained to jump variable short distances up to $25 \mathrm{~cm}$ to a moving $7 \times 10-\mathrm{cm}$ elevated platform, like the stationary one, mounted on a turntable that rotated once every $2 \mathrm{sec}$. The outer edge of the platform, about $17 \mathrm{~cm}$ from the turntable center, traveled about 50 $\mathrm{cm} / \mathrm{sec}$, but was made slower as needed. The rats first stepped short distances, then jumped longer distances to the platform, usually as it passed nearest to them but sometimes variously at more distant points. Although other methods were explored, the rat's natural tendency to step or jump onto the moving platform was the motivation used.

Visual Pattern Discrimination. Two rats operated on as infants and three as adults, all a year old, four animals operated on as infants 7 months old, and five normal sibs from the three corresponding litters were trained to discriminate visually between the two members of pairs of a series of patterns. An additional animal hemispherectomized as an infant and two of its normal sibs were trained beginning in the third week of life on the pattern discrimination and several additional visual tasks. The apparatus, on a table in a corner, was similar to that originally described by Lashley (17): two square doorways $15 \mathrm{~cm}$ on a side, $5 \mathrm{~cm}$ apart with their sills $24 \mathrm{~cm}$ above a table, were centered in a vertical board $60 \times 60$ $\mathrm{cm}$. Cards cut from light poster board or other material bearing the patterns served as doors and fitted loosely in the doorways. They could be locked or held in place. On the center rear of a platform $60 \times 30-\mathrm{cm}$ deep behind the doors, a piece of food pellet and water were available for food-deprived subjects. The apparatus and surroundings were flat black. Normally one wall was on the animals' left, the other beyond the platform, as they faced the cards. They were trained to jump from the round platform mentioned earlier directly through or at the center of the opening, with the cards in place, making unequivocal decisions. They were placed on the jumping platform facing away from the doors. No deflectors were used. Jumping distances were $12-16 \mathrm{~cm}$; longer distances were not used because they seemed to generate erratic and escape behavior.

The patterns, mostly white figures on black background, were stripes, triangles, and segments of triangles. Black and white alternating stripes 
were $1.8 \mathrm{~cm}$ wide, outlines of triangles $1 \mathrm{~cm}$ wide. The standard triangle was outline and $12 \mathrm{~cm}$ on a side: a solid standard-size triangle was also used. In some instances a gray standard-size outline triangle and two smaller white outline triangles 10 or $8 \mathrm{~cm}$ on a side were paired with the standard white outline triangle. The triangle fractions, made on the same template as the standard outline triangle, were the base only, the right side, the right side and base, a small part of the apex, and the right side and apex. The figures were usually Mystik tape applied to the posterboard, but Marks-a-lot ink and paints were sometimes used. Standard outline triangles to be viewed by transmitted light were made with opaque tape and translucent tracing paper applied to the back side of "cards" of clear Plexiglas $1 / 16$ inch thick.

Under food deprivation, as mentioned earlier, the adult animals in this group were accustomed to going through the doors, then given daily sessions of about 20-30 trials, first with a black and a white card as a pair, then pairs of striped cards, standard triangles, solid triangles, and segments of triangles. Jumping to the white card, the vertical stripes, upright triangle, and upright triangle segments which were designated positive, represented correct responses. Horizontal stripes, inverted triangles, and segments of inverted triangles were negative. One card in positive position was presented randomly in one or the other doorway once to four times in succession with the other card locked or held in the other doorway in negative position. Criterion for black and white, stripes, and standard triangles was 20 successive errorless trials in one session. A novel set of trials with the solid triangles followed with both doors unlocked, the observer and the rat separated from each other's view by a screen, when the rat was on the jumping stand. A second observer who had not trained the rat repeated this procedure. The segments of triangles were then presented, each pair usually for a single session of 20-25 trials, both doors unlocked. One error (or none) in 20 consecutive trials was considered evidence of discrimination; two errors, a common score on some segments, suggested equivalence. (Rats that discriminated the small apex considered the inverted figure positive.)

The young rats begun in their third week on pattern discrimination went through the schedule just outlined and were also tested with the gray and white, unequal size, and translucent triangles made of Plexiglas. As noted, for the young rats exploration, handling, and other aspects of the procedure provided the reinforcement.

Vision in the eye opposite the hemispherectomy was tested in some animals with the other eye closed, after the animal had already learned with both eyes open.

Controls for Nonvisual Cues. In pattern discrimination schedules, multiple sets of cards of different materials, several patterns, random exchange 
of the pairs of cards between the doors, sessions with both doors unlocked to provide no differential reinforcement, and no difference in acoustical cues between the doors were intended to provide evidence that the rats were attending primarily to visual details of the patterns $(5,25)$.

A procedure applied to some animals in the present experiment, and to others, at the close of the visual pattern discrimination schedule was to conduct trials with the Lashley apparatus in its normal position with the animals' eyes closed, then again with them open. An additional part of the procedure was also to give the animal an opportunity to jump through the doors when the whole apparatus was rotated $180^{\circ}$ in relation to the walls. Eyelids were closed with sutures, and sutures removed, under ether anesthesia. Testing was done 8-20 hr after anesthesia. Additional experiments to assess nonvisual cues were observations of rats whose eyes or middle ear contents were removed at birth in operations done under ether anesthesia. The behavior of animals with eyes closed, and blind or deaf animals is described under Results.

Morphologic Studies. Brains and spinal cords, divided into blocks, and eyes in situ in the skull base were usually fixed in Bouin's fluid made with $10 \%$ formalin, paraffin embedded, and section $8 \mu$ thick stained with Luxol fast blue and cresyl violet. Most brains were completely serially sectioned, the others examined by representative or serial sections of the blocks.

Mentioned earlier were the hemispherectomized and normal rats used for study of the corticospinal tract by the Fink-Heimer-Nauta procedure. Three normal mature rats 13,9 , and 2 months old had the dorsal frontal cortex removed on one side; two rats hemispherectomized as newborns had the dorsal frontal cortex of the remaining hemisphere removed at ages 8 and 9 months; and two rats hemispherectomized at $6 \mathrm{~T} / 2$ weeks of age had the dorsal frontal cortex of the remaining hemisphere removed when they were 13 months old (the normal rat 13 months old was their littermate). Another normal rat 10 weeks old was hemispherectomized. The animals were killed 5 days after the ablations except the rat 2 months old which was killed at 3 days. Five infant rats aboout 20 or $40 \mathrm{hr}$ old had the dorsal frontal cortex on one side removed and were killed 11-15 hr later. The latter interval was optimal because disintegration of the fibers and growth in size of the animal are rapid, and longer intervals work to dilute the fiber fragments beyond useful recognition. Staining was applied to sections at intervals chiefly in the medulla and spinal cord, but higher levels were also surveyed.

\section{Results}

Motor-sensory Functions and General Observations. Rats hemispherectomized as infants: These differed little from their normal littermates func- 
tionally until the seventeenth day, when certain placing reactions disappeared, as described later. The cranium appeared smaller than normal in the second week but this was not noticeable later. As they matured, the hemispherectomized rats were almost always smaller then their normal sibs, about $10-20 \%$ less in weight. From hours onward after operation response to pinprick over the body, crawling, hopping and stepping reactions, righting, stopping at a precipice, and crowding to nurse appeared normal. Juvenile romping and sham fighting resembled the normal, but with the passage of months, the animals operated on became less active than normals in home cages, though during behavioral tests they were active and responsive. $A$ few animals were startled by being approached and touched on the side opposite the ablation. Some occasionally harmlessly bit their limbs on that side; normal rats rarely behaved comparably.

Lateral tactile placing with the forefoot opposite the hemispherectomy disappeared permanently during the seventeenth day, as did the corresponding visual placing, and, as well as could be determined, tactile placing with the hind limb. In one litter with four hemispherectomized subjects observed at close intervals over a 20 -hr period during the seventeenth day, the placing became less and less likely to be elicited and stopped almost simultaneously among the four. With the loss, the animal now let the affected limbs slip repeatedly over the runway on contact with the edge, usually quickly retrieving them. This interfered with walking or running on the edge, but in ensuing weeks the slipping improved to involve just the toes and sometimes feet. When standing, the animals showed a tendency at times to a slightly swayback posture, that is, less than the normal dorsal arching of the back from scapulae to rump. This disappeared in running. There was also a tendency sometimes for the affected forefoot to be slightly flexed at rest, but no other abnormality or awkwardness of movement of the limb was observed.

The rats hemispherectomized as infants neither tactually placed (laterally) nor visually placed with the forelimb and eye opposite the lesion, but, as noted, both stimuli together elicited placing in some animals. Some animals when allowed to turn their heads and view a ledge on the side opposite the ablation, using the eye on the same side as the lesion, placed with the affected limb. A commoner response in this circumstance was to carry the unaffected forefoot across on the other side and place it on the ledge. In a few instances, both feet were placed.

The two subjects with left hemispherectomy behaved as animals with the lesion on the right, except that the abnormal functions were transposed.

On a level flat surface, locomotion of these animals appeared similar to that of normals, and only when the affected feet contacted the edges was abnormality evident. Analysis of the slow-motion pictures showed little difference between the animals operated on as infants and normals, except the 
animals who had simultaneous ablation of the remaining frontal cortex. In the next section, locomotion of the animals hemispherectomized as infants and adults and normal animals are compared.

Rats hemispherectomized as adults: In most respects these animals were indistinguishable from those operated on in infancy. For 2 or 3 days after operation, however, they were severely incapacitated, scarcely able to stand, sometimes thrashing briefly when startled. For a few days more they fell off the runway if the affected feet slipped over the edge, though they could now walk and run on the flat. They rapidly improved to resemble those operated on in infancy. The animals operated on at $6 \frac{1}{2}$ weeks, but not the others, tactually placed fairly constantly with the hind foot opposite the ablation. All those hemispherectomized as adults placed visually with the eye and forelimb opposite the lesion up to about 10 days after operation, then this response ceased. Combined visual and tactile placing or crossplacing with the other eye continued.

Motion pictures of locomotion of the rats operated on as infants and as adults on horizontal ladders showed only that they ran well on them, making no more slips and stumbling no more than normals.

In analysis of slow-motion pictures of rats with cerebellar abnormalities or lucal cortical ablations running on the hat, it had been found useful to attend to the successive positions of the limbs during their cycles of motion, the coordination of these with each other, the posture of the body axis, tail, and head, and movements of the pelvis (13). Locomotion on the runway was mostly walking, running at a moderate rate, or running fast; in the running cycle the alternate fore- and hind foot were on ground almost simultaneously. "Galloping" was rare and is not considered. Among the animals operated on as adults, the pelvis swung from side to side and rotated on the long axis of the body a little more than in normals, during slower locomotion, but in faster running this was not noticeable. Subjects operated on in infancy did not show this. Frequent, spontaneous runs of 1 or 2 meters and sometimes the length of the runway, among many short runs, was usual normal behavior up to about 4 months. Older animals ran much less enthusiastically. Running fast, a normal rat 17-25 days old traveled about $50-60 \mathrm{~cm} / \mathrm{sec}$ with a stride of about $11 \mathrm{~cm}$; at $7-12$ weeks $60-80 \mathrm{~cm} / \mathrm{sec}$, and about an $18-\mathrm{cm}$ stride. A characteristic of fast running in the normal rat, first appearing at 17 days of age, was a distinctive forward reaching thrust of the forelimb, almost horizontal, at the end of which the foot began its descent to the runway surface. Just at the extreme of this thrust the toes were hyperextended and spread apart with the fifth digit lowest, such that the tip of that digit and the metatarsal pads first touched ground. In slower locomotion, the thrust was inclined downward. In the rats operated on in infancy, except those with the combined 
ablations, this aspect of gait was virtually indistinguishable from normal during moderate to fast running. In the animals hemispherectomized as adults this "high-stepping" thrust was not fully developed even in fastest running, and in consequence, the animals' stride (conveniently measured in the forelimb) was shorter, by 2 or $3 \mathrm{~cm}$ at 11 weeks, for example. The two rats with combined ablations also had impairment of the stride thrust components. One rat operated on as an adult differed from the others in never running fast or as smoothly as the others.

$V$ isually Guided Jumping. All the hemispherectomized rats could jump variable distances from one platform to another, gauging the distances as well as normals. Most could jump $40 \mathrm{~cm}$, the maximum usually tested in adults. Both normal and abnormal animals tended to undershoot the mark slightly when the distance was substantially increased, and overshoot when it was reduced. This amounted to landing toward the near or far end of the small landing platform; it was usually minor and corrected on the next jump if the distance was not changed. Two rats operated on as infants regularly took off from one side of the jumping platform and landed toward the opposite side of the landing platform, but were otherwise accurate. As on the runway, the animals had some trouble with their affected feet slipping off the edge of the round jumping platform and rarely they fell off. There was little evidence of slipping on the landing platform; the precision in jumping, as observed in movies, was essentially similar to that of normals. All hemispherectomized rats waved the affected forelimb up and down in space as they set themselves to jump.

Several subjects operated on as infants or adults jumped accurately and as well as normals variable distances up to 25 or $30 \mathrm{~cm}$ to the small lighted platform set at the dimmest in the dark.

The optic nerve on the side of the hemispherectomy was intact in all animals except one that was operated on as an infant, in which it was cut through. Vision was limited in this rat from the beginning to the eye opposite the ablation which was thus only served by uncrossed retinogeniculate fibers. It jumped accurately up to $25 \mathrm{~cm}$ but wauld not jump farther. The rat hemispherectomized at birth that was trained from the third week on all of the visual tasks. using both eyes, was then also tested with only the eye opposite the ablation. It was able to jump variable distances accurately to the $7 \times 10-\mathrm{cm}$ platform placed in different positions with different backgrounds and relationships to walls. It jumped to the dimly lighted platform in the dark up to $30 \mathrm{~cm}$, and $10-20 \mathrm{~cm}$ (out of vibrissae reach) to the moving platform.

Visual Pattern Discrimination. The ten subjects that were tested (all had right hemispherectomy) learned to discriminate the patterns to criterion as described in Methods. One of those operated on as an adult and 
the seven hemispherectomized as infants were also tested on triangles with the right eye closed: the scores of the former and two of the latter were at chance level. Four of the other five of those operated on as infants performed to criterion in a single session; the fifth made three errors in 40 trials. The eye on the side of the hemispherectomy, served by crossed fibers, was not tested differentially except that all operates, save the one noted, placed visually with that eye.

The rat hemispherectomized as an infant and trained from the third week on all of the visual tasks, using both eyes, discriminated the transmitted-light triangles and was not confused when one of the triangles was gray or when it was smaller than its standard paired member, reaching the criterion for these tests of one error in 20 consecutive trials in a single session with doors unlocked. It then discriminated just as successfully on these same tests using only the left eye.

The number of trials to criterion in the discrimination of stripes and triangles varied among the hemispherectomized rats and their controls trained as adults, as it did in other experimental groups. After introduction to the apparatus for a couple of sessions, trials on black and white were given. The five normals attained criterion in two or three sessions, those operated on in three to six. Normals attained criterion on stripes in two to six sessions, that is, about 50-130 trials, hemispherectomized subjects in three to five sessions. Normals required three to five sessions for triangles, animals operated on, three to ten sessions; among the latter, one hemispherectomized as an adult reached criterion in four sessions, and two operated as infants reached it in three and four sessions, respectively. The animal with one of the most nearly complete hemispherectomies represented in Fig. 1 reached criterion on black and white, stripes, and triangles in three, three, and four sessions, respectively. Review of these scores in unusual detail is given to show that in the setting of this experiment some hemispherectomized subjects were capable of learning about as rapidly as the best normals.

Controls for Nonvisual Cues. The different kinds of cards and their presentations were noted in Methods. One rat operated on as an infant that discriminated with the left eye, and one that failed to were tested with both eyes closed and then reopened as noted. We describe their behavior together with that of 29 other normal rats and rats with various visual system defects that have since been similarly observed, because it supports the conclusion that the animal was using visual cues primarily in the pattern discrimination. In their accustomed setting, the temporarily sightless animals jumped through the doors much as they did with eyes open, but their discrimination scores dropped to chance levels. If the whole apparatus was rotated $180^{\circ}$, some rats continued to jump into space toward where the doors would normally have been, others searched in all directions but did 
not jump. Only if the jumping platform was close enough to the doors to permit vibrissae contact with them would they jump through the doors in their abnormal position. With eyes open again in the normal setting, discrimination immediately returned to none or one error in 20 trials. With eyes open, too, the rats would jump through the doors when the apparatus was in unusual situations.

The four rats with middle ear contents removed in infancy, but with vibrissae intact, did not alert or orient to common sounds or a $300-\mathrm{Hz}$ pure tone pulse which normals did, but they sometimes startled at a loud finger snap or electric cord switch $30 \mathrm{~cm}$ away but hidden from their view. They learned to discriminate stripes and triangles to criterion, and to jump to the stationary and moving platforms, and to the dimly lighted platform in the dark, as well as normals.

The four rats made blind at birth, but with ears and vibrissae intact, learned by small increments to jump first on the large platforms to $20 \mathrm{~cm}$ by the age of 5 weeks, then from the round to the small platform by 2 months. (As in the other experiments with young animals, reinforcement was exploration, jumping, and being handled.) The general arrangement of the platforms and cushions on a table with a wall on the animal's left as it prepared to jump was kept uniform during the earlier training. Once accustomed to this activity, they could usually adjust their jumps to land on the platform from jumping positions $15 \mathrm{~cm}$ on either side of the normal position, and land on the near end of the platform from $30 \mathrm{~cm}$. If the landing platform was moved $15 \mathrm{~cm}$ to either side of its normal position, or taken away, they jumped into space to where it would normally be. If the whole arrangement was rotated $180^{\circ}$ in relation to the wall, the animal jumped $180^{\circ}$ away from the landing platform. One rat, an enthusiastic jumper at 10 weeks of age, could locate the landing platform fairly accurately $25 \mathrm{~cm}$ away in unusual relation to surrounding walls if the platform was first placed within tactual reach a few times then moved away stepwise up to $25 \mathrm{~cm}$ in a constant linear direction. In contrast to the blind rats, the normal sibs jumped accurately to the platform under almost any circumstance, from the experimenter's hand, and back and forth on the platforms.

The blind rats readily learned to go through the doors of the Lashley apparatus, pushing the cards down vigorously but randomly in respect to the patterns. They switched very quickly from a locked door to the other; they jumped to the doors only as far as they could first reach with vibrissae. Scores were at chance levels during three weeks of daily sessions. They stepped onto the moving platform likc other rats, but only if they happened to contact it as it came within a couple of centimeters of the jumping platform.

Morphologic Studies. The extent of the hemispherectomy operations was 
given in Methods. Among the secondary gross consequences of the ablation was a moderate displacement of the residual brain toward the operated side in those operated on as infants, little in those hemispherectomized as adults. The corticospinal tract corresponding to the lesion was absent except for the infant "remodeling" as noted later. In subjects operated on as infants the medial lemniscus persisted in a trace almost as far rostral as the ablation, and it was considerably reduced retrograde as far as its source. The contralateral cuneate and gracile nuclei, and their arcuate fibers, were reduced by about a third to a half of their usual volume. In those hemispherectomized as adults the reduction of the tract and nuclei was slight. Evidence of transneuronal degeneration in the pontine nuclei could not be detected, but the absent corticospinal tract made comparison of the two sides difficult. In all subjects there was an unexplained moderate to marked loss of lateral lemniscus fibers in their distal course toward the inferior colliculus, but proximally and in the trapezoid body complex no alteration was recognized. The optic nerve opposite the ablation in all animals, except the one with the considerable residual thalamus, was greatly reduced in size, but more so in those operated upon in infancy. The accessory optic fiber systems were obliterated on the side of the ablation, except in one animal in which a few fibers, perhaps nonoptic components, coursed adjacent to the region of the nucleus of the accessory optic tract. The fornix was absent and depending on what midline structures were incompletely removed some fibers of the habenulointerpeduncular and mammillothalamic tracts sometimes persisted. The mammilotegmental tract was rudimentary or absent, and the dorsal tegmental nucleus was variably smaller than normal on the side of the ablation in animals operated on as infants. Evidence of effects in the cerebellum was not recognized.

Characteristically there was no gliosis at the margins of the brain injuries made in infancy (13) or evidence of old hemorrhage, and except for the slight gross expansion mentioned and occasional locally aberrant courses of some midline fiber systems such as the posterior commissure, the defects looked almost like artifacts compared with lesions incurred in adult life. In the margins of the ablations of the brain made in adults there was minimal gliosis and some hemosiderin-containing phagocytes in places in the wound margins, the latter giving a brown tinge to some parts grossly.

Ganglion cells in the retina were reduced in number in the eye opposite the ablations made in adults and infants. Qualitatively the deficit seemed to be spread widely over the retina, but no quantitative determinations were made.

In the three normal rats whose frontal cortex was removed on one side and the fourth in whom hemispherectomy was performed several days be- 
fore they were killed, the Fink-Heimer-Nauta stain showed abundant degenerating fibers along the corticospinal tract which crossed completely in the decussation in the medulla and descended in the posterior funiculus in the other side for the length of the spinal cord, a few fibers persisting as far as the sacral part. As they coursed toward the other side in the decussation, numerous fibers departed from the decussating bundles and gathering corticospinal tract to enter the caudal domains of the gracile and cuneate nuclei, the caudal spinal trigeminal nucleus, medially situated nuclei in the dorsal gray near the central canal (but not the hypoglossal), and the reticular region more laterally $(1,18,31)$. Fewer fibers penetrated the ventral gray matter. Decussating corticospinal tract fibers traveled with those in the internal arcuate fiber bundles where the two systems were close to each other. In the spinal cord, fibers from the tract entered the dorsal gray matter, fragments of axons being most abundant in the medial gray matter abutting the tract. Relatively few entered the ventral gray matter. These ramifications diminished progressively from the cervical region caudalward. The hemispherectomized animal was included in this part of the study to determine whether there might be uncrossed corticospinal fibers from other parts of the cortex of hemisphere not revealed by the usual frontal cortical ablations. As noted it showed that there was none. It also showed that the only degenerating fibers that coursed beyond the medulla into the spinal cord were those in the domain of the corticospinal tract.

In the rats hemispherectomized at maturity, the old degeneration in the corticospinal tract appeared as scattered, mostly coarse, black granules, large enough to he seen in the photomicrograph at low magnification in Fig. 2. The granules rostral to the decussation were the same as they were caudal. In size, shape, and distribution they corresponded to some of the persistent fiber remnants that stained with Luxol fast blue in the same situations in nearby sections. These remnants, that had persisted for a year, characterized this kind of fiber degeneration in other systems such as the optic tract fibers in the midbrain on the side of the hemispherectomy. Nowhere in these tracts were there degenerated fibers recognizable as the finely segmented or beaded black lines so characteristic of the staining when it is applied soon after the fibers have begun to degenerate. Thus there was no evidence of the formation of uncrossed corticospinal fibers after the hemispherectomy in the mature animal.

In the animals operated on as infants and studied with the Fink Heimer-Nauta procedure after ablation of the frontal cortex in the remaining hemisphere when they were adults, there was no residuum of corticospinal tract corresponding to the hemisphercetomy. The tiny fibers of infancy would be diluted beyond ready recognition by the stretching and 


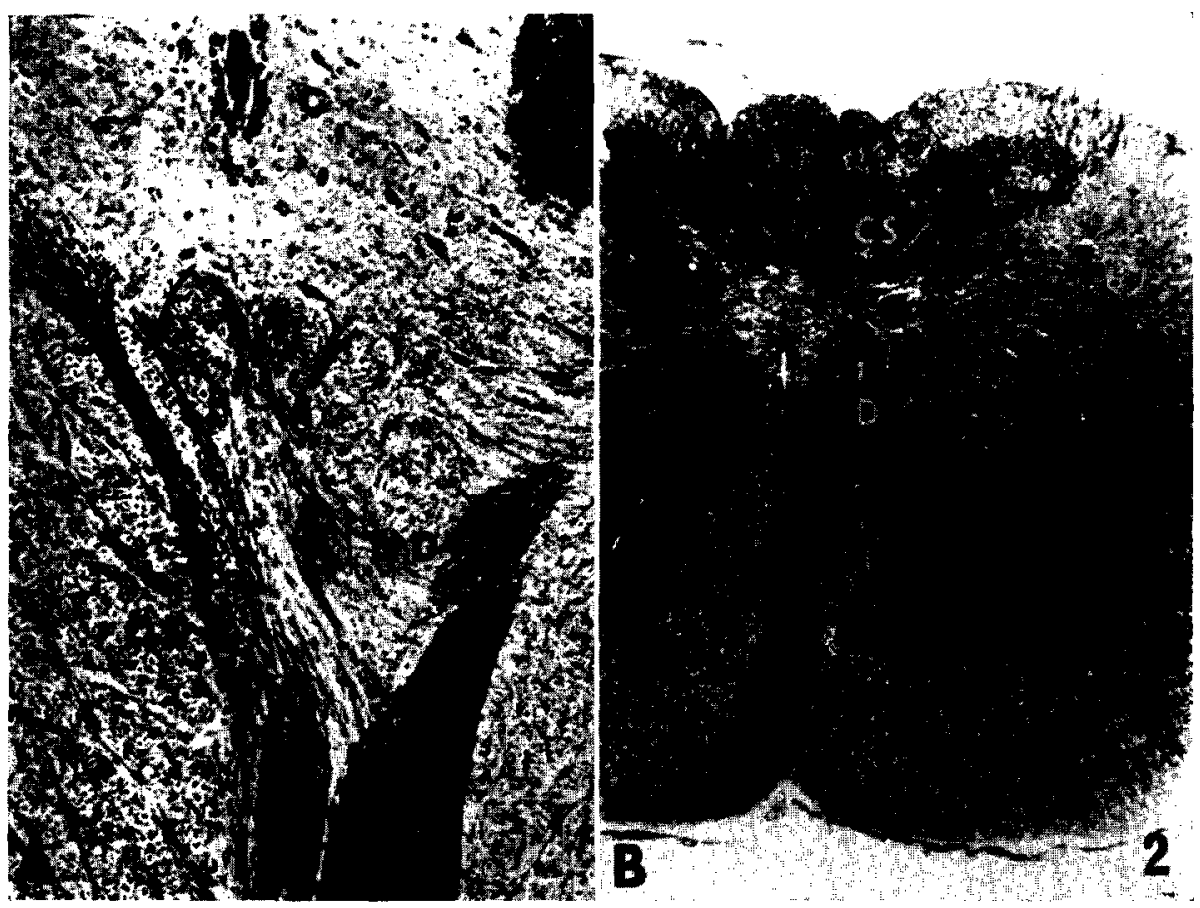

FIG. 2. A. Center of the corticospinal tract decussation, frontal section, in a rat hemispherectomized at $6 \frac{1}{2}$ weeks of age and killed 13 months later. Five days before it was killed the dorsal frontal cortex on the remaining hemisphere was ablated. The old degeneration, OD, from the hemispherectomy shows as coarse granules, the new degeneration, ND, from the cortical ablation is fine-grained. Both tracts crossed completely. The central canal is at top near center (Fink-Heimer-Nauta stain, $\times 70$ ). B. Decussation, D, of the remaining corticospinal tract, frontal section, in a rat hemispherectomized at birth. Five days before it was killed at age 8 months, the dorsal frontal cortex on the remaining hemisphere was ablated. Most of the tract decussates, $D$, and the gathering fibers of the corticuspinal tract, CS, approach the dorsal funiculus. Arrows indicate bundles, barely visible at this magnification, containing uncrossed aberrant fibers. There were no residual corticospinal tract fibers corresponding to the hemispherectomy. The rectangles $\mathrm{A}$ and $\mathrm{B}$ are magnified in Fig. 3 (Fink-HeimerNauta stain, $\times 20$ ).

expansion incurred in the growth in size of the animal. However, the degeneration corresponding to the frontal cortical ablation done in the remaining hemisphere differed from that in the rats hemispherectomized as adults. A form of remodeling had occurred. There was formation of a sparse uncrossed corticospinal tract as well as the regular crossed corticospinal tract (Figs. 2-4). The uncrossed fibers and their terminations were relatively most abundant in the medulla at the levels of the decussation corresponding to the normal distribution. Their number dwindled progres- 


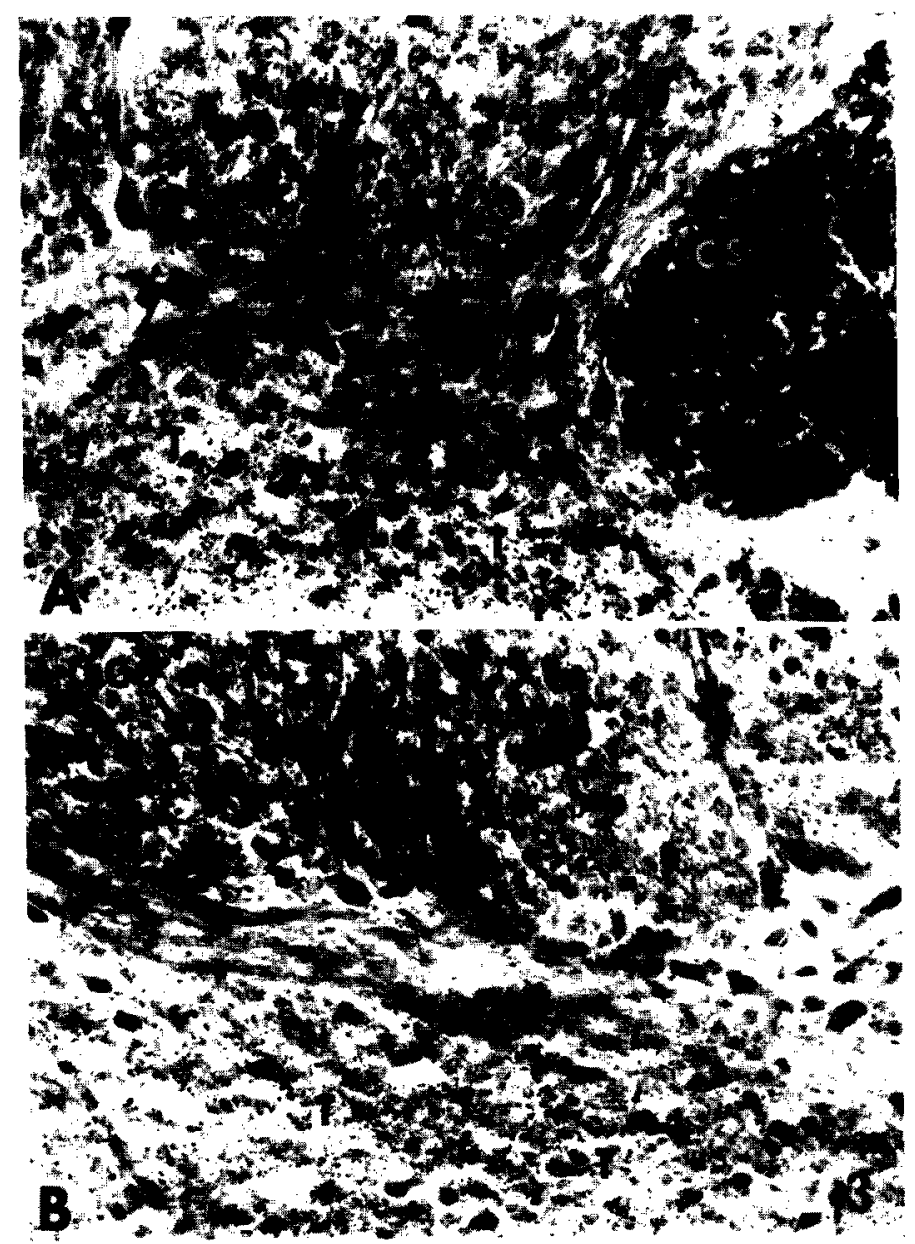

FIG. 3. The rectangles $\mathrm{A}$ and $\mathrm{B}$ in Fig. $2 \mathrm{~B}$ are magnified. $\mathrm{A}$ is the right side with midline at the left margin showing the gathering corticospinal tract, CS, approaching the dorsal funiculus. Abundant terminating fibers, $T$, penetrate the adjacent dorsomedial gray matter. $B$ is the left side, with the midline at the right margin, showing scattered fibers of the gathering uncrossed corticospinal tract, UCS, and fairly numerous terminating fibers, $T$, penetrating the adjacent dorsomedial gray matter (Fink-Heimer-Nauta stain, $\times 200)$.

sively from the cervical region (Fig. 4) caudalward. A very few fibers were traced as far as the lumbar cord. Small bundles of fibers turned off from the main decussating tract to course dorsalward on the same side in which the tract had descended in the brainstem. They took pathways and formed bundles in miniature that scemed to be essentially mirror images of the normal crossed fibers. Numerous fine black grains and short lengths of 


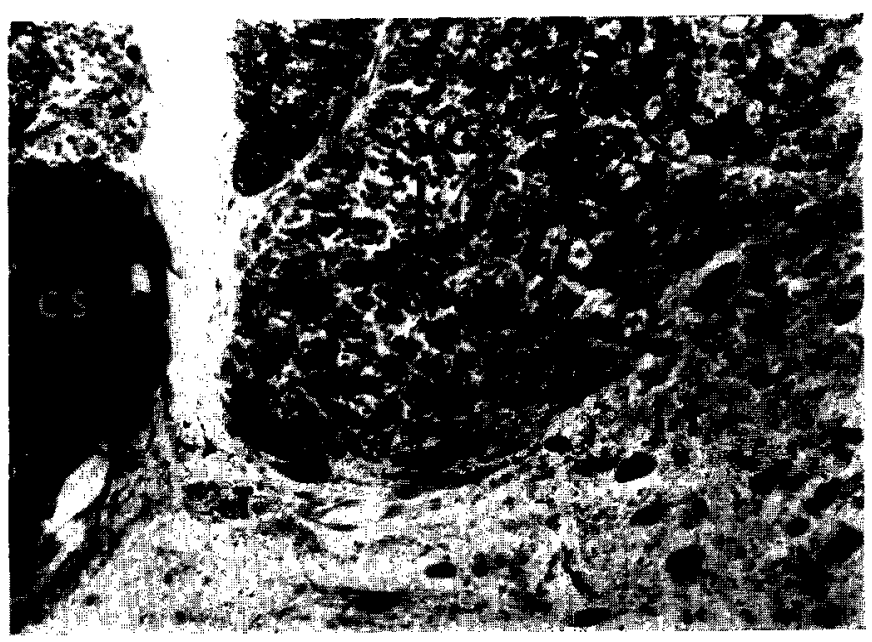

FIG. 4. A portion of the dorsomedial part of the cervical spinal cord, frontal section, from the rat represented in Figs. $2 \mathrm{~B}$ and 3, but right and left are transposed. The medial part of the crossed corticospinal tract, $\mathrm{CS}$, and the scattered fibers of the uncrossed aberrant corticospinal tract, UCS, are shown. $T$ indicates terminating fibers penetrating the adjacent dorsomedial gray matter (Fink-Heimer-Nauta stain, $\times 200$ ).

segmented or beaded fibers were present in the midline and medial dorsal gray matter on the "wrong" side, fewer more laterally, but almost none in the ventral gray matter. The distribution, though the fibers were much fewer, corresponded to the normal terminations in medial, gracile, cuneate, spinal trigeminal and reticular nuclei of the caudal medulla. In the spinal cord, especially the cervical, short segments of degenerating fibers rarely crossed the midline horizontally from the normal crossed tract to the ventralmost part of the other dorsal funiculus or its adjacent gray matter close to the midline. Throughout the cord black grains and occasional segments of fibers were present in the medial dorsal gray column (medial part of the intermediate nucleus) adjacent to the aberrant tract. Very few extended more laterally. Corticospinal tract fibers had reached the decussation and the rostral part of the cervical spinal cord by the time of birth. Whether the uncrossed fibers arose by branching of axons (10) already in the decussation at the time of birth, or by diversion later on of fibers that had not yet descended to the medulla, is not known.

The corticospinal tract in the rat is relatively huge with a large part terminating in the dorsal gray matter of the caudal medulla as noted. The uncrossed aberrant path here was very small and unimpressive compared to the big normal tract, yet the total number of fibers distributed to the caudal gray matter of the medulla, especially dorsomedially, was still large. 


\section{Discussion}

The relatively sudden disappearance of the tactile placing reaction during the seventeenth day in the rats hemispherectomized as infants, a reaction that was indistinguishable from the normal up until that time, remains a puzzle. Degeneration studies around the seventeenth day to show the changing developmental state of the medial lemniscus, corticospinal tracts, and ascending noncerebellar path in the lateral funiculus of the spinal cord said to be essential to tactile placing (21) may provide clues that conventional histologic studies have not.

In all of the hemispherectomized rats but one, visual tasks had been first learned using the eye of the side of the hemispherectomy, as well as the eye with only uncrossed fibers if it was functioning. It was useful, nonetheless, to find out that the uncrossed fibers alone in the presence of hemispherectomy could mediate detail vision. The functional capacity of the uncrossed optic fibers in the rat had been studied earlier by several investigators by occluding vision in one eye and removing the visual cortex on the same side $(16,22)$. In this arrangement the open eye was presumed to be subserved only by uncrossed fibers, and visual cortex was defined as that part of the posterior dorsal cortex which when wholly removed resulted in degeneration of all neurons in the corresponding dorsal lateral geniculate nucleus. Recent experiments have shown that the rat, like some other mammals, retains considerable detail vision after removal of this visual cortex, though the mechanisms for the vision are unknown (19). This raises the question whether the rats in the other experiments $(16,22)$ were using only uncrossed retinofugal pathways. The hemispherectomy experiments assured that only the uncrossed pathways were available.

Comparison is difficult, but some other experimental animals more deprived than the hemispherectomized rats illustrate how much the visual apparatus can be reduced and still maintain useful vision. Cats with less than $2 \%$ of their optic tract fibers carried out a variety of visual tasks (9). Rats whose retinas were extremely malformed and deficient from irradiation or whose visual cortices and superior colliculi were extensively damaged at birth, grew up with considerable ability to discriminate patterns and jump variable distances and directions to platforms as described in Methods (12).

Finding a form of "morphologic plasticity," the development of uncrossed corticospinal tract fibers, in the rats hemispherectomized in infancy led us to look for functional differences between these animals and those operated on at maturity that might be related to corticospinal tract functions. Impairment of the forelimb stride component of running in the latter 
animals was the principal difference found. Experiments done in preparation for the hemispherectomy work helped to assess it. In substance, these were morphologic and functional studies of 24 rats from whom the frontal or frontal-dorsal cortex was ablated unilaterally or sometimes bilaterally in early infant, juvenile, or adult stages. They showed that the more rostral lesions caused some degeneration of the corticospinal tract without affecting tactile placing. Lesions involving the more posterior part of the motor-sensory cortex, a few of which encroached on the somatosensory area I (30), were necessary to impair or abolish placing, but they also diminished the corticospinal tract. Both kinds of lesions impaired the forward thrust forelimb stride component of running if they were made after the age of 16 days. Except for one rat with extensive bilateral ablations made in early infancy which showed some impairmant of stride, those operated on in infancy were spared. Two conclusions seemed possible. (a) The stride feature of running was an expression of corticospinal tract activity and it was at least partly dissociated from the placing reaction essential for positioning the feet while running on "edges" on rough terrain. (b) There was a sparing effect when the lesions were made in infancy, unless the lesions were quite extensive. This seemed to be borne out by the diminished stride of the two rats with combined hemispherectomy and frontal ablation in infancy.

The dissociation of stride and placing, together with sparing of stride in animals hemispherectomized in infancy, suggested that the uncrossed corticospinal tract fibers provided the bilateral corticospinal innervation essential for normal stride. At present there is no proof beyond the coincidence of the remodeling and the sparing that the aberrant corticospinal tract fibers performed any useful function. The corticospinal tract system from rats to primates seems to influence motion chiefly by way of afferent systems where most of its terminations lie. In some studies its cells of origin and fiber destinations appear to be laid out in somatotopic fashion, but from other views it appears more cryptically organized to influence integrated patterns of mation $(1,2,4,18,27,31)$. The kind of injury used in experiments to determine whether the mechanism for stride in running is somatotopically represented in the nervous system, or organized in some other way, complicates the assessment. Our hemispherectomized animals showed little else than disordered placing and stride, but in experiments by other investigators in which localized frontal cortical lesions were placed (31) and in those of our own in which cortical ablations encroached on somatosensory cortex, much more severe and localized impairment of limb functions occurred. Considerably more needs to be known about the organization of the rat's corticospinal tract and related systems, including those 
governing placing and positioning the limbs in motion, before the remodeled tract can be well evaluated.

\section{References}

1. Barnard, J. W., and C. N. Woolsey. 1956. A study of localization in the corticospinal tracts of monkey and rat. J. Comp. Neurol. 105 : 25-50.

2. BARron, D. H. 1934. The results of unilateral pyramidal section in the rat. J. Comp. Neurol. 60: 45-55.

3. Boyd, J. D. 1970. Cited in Hicks, S. P., Embryogenic disorders of the nervous system; In "The Cellular and Molecular Basis of Neurologic Disease." G. M. Shy, E. S. Goldensohn, and S. H. Appel [eds.]. Lea and Febiger, Philadelphia (in press).

4. Bucy, P. C. 1966. The delusion of the obvious. Ferspect. Biol. Med. 9: 358-368.

5. Dodwell, R. C., and N. L. Freedman. 1968. Visual form discrimination after removal of the visual cortex in cats. Science $160: 559-560$.

6. Doty, R. W. 1953. Effects of ablations of visual cortex in neonatal and adult cats, pp. 316-7. In "Abstracts of Communications, XIX International Physiological Congress" Therien Freres, Ltd., Montreal.

7. Fink, R. P., and L. Heimer. 1967. Two methods for selective silver impregnation of degenerating axons and their synaptic endings in the central nervous system. Brain Res. 4 : 369-374.

8. FrenCH, L. A., and D. R. Johnson. 1955. Examination of the sensory system in patients after hemispherectomy. Neurology $5: 390393$.

9. Galambos, R., T. T. Norton, and G. P. Frommel. 1967. Optic tract lesions sparing pattern vision in cats. Exp. Neurol. 18: 8-25.

10. Goodman, D. C., and J. A. Horel. 1966. Sprouting of optic tract projections in the brain stem of the rat. J. Comp. Neurol. $127: 71-88$.

11. Hicks, S. P., and C. J. D'Amato. 1966. Effects of ionizing radiations on mammalian development, pp. 195-250. In "Advances in Teratology." D. H. M. Woollam [ed]. Logos Press, Ltd., London.

12. Hicks, S. P. 1970. Effects of radiation and other factors on the developing form and function of the nervous system. USAEC, Div. Tech. Inform. Ext., Nuc. Sc. Abst. No. C00-1201-28.

13. Hicks, S. P., C. J. D’Amato, S. J. Klein, L. L. Austin, and B. C. French. 1969. Effects of regional irradiation or ablation of the infant rat cerebellum on motor development, pp. 739-754. In "Radiation Biology of the Fetal and Juvenile Mammal, 9th Annual Hanford Biology Symposium." USAEC, Div. Tech. Inform. Ext., Oak Ridge, Tenn.

14. Isaacson, R. L., A. J. Nonneman, and L. W. Schmalz. 1963. Behavioral and anatomic sequelae of damage to the infant limbic system, pp. 41-78. In "The Neuropsychology of Development." R. L. Isaacson [ed.]. Wiley, New York.

15. Kennard, M. A. 1942. Cortical reorganization of motor function: studies on a series of monkeys of various ages from infancy to maturity. A.M.A. Arch. Neurol. Psychiat. 8 : 227-240.

16. Lashley, K. S. 1924. Studies of cerebral function in learning. VI. The theory that synaptic resistance is reduced by the passage of the nerve impulse. Psychol. Rev. $31: 369-375$. 
17. LASHLEY, K. S. 1930. The mechanisms of vision. I. A method for rapid analysis of pattern-vision in the rat. J. Genet. Psychol. $37: 453-460$.

18. Lawrence, D. G., and H. G. J. M. Kuypers. 1968. The functional organization of the motor system in the monkey. I. The effects of bilateral pyramidal lesions. Brain 91 : $1-36$.

19. Lewellyn, D., G. Lowes, and R. L. IsaAcson. 1969. Visually mediated behaviors following neocortical destruction in the rat. J. Comp. Physiol. Psychol. 69: 25-52.

20. Lund, R. D. 1965. Uncrossed visual pathways of hooded and albino rats. Science 149 : 1506.

21. Lundberg, A., and U. Norsell. 1960. Spinal afferent pathway of the tactile placing reaction. Experientia $16: 123$.

22. Muntz, W. R. A., and N. S. Sutherland. 1964. The role of crossed and uncrossed optic nerve fibers in the visual discrimination of shape by rats. $J$. Comp. Neurol. 122 : 69-77.

23. Nathan, P. W, and M. C. Smith. 1950. Normal mentality associated with a maldeveloped "rhinencephalon." J. Neurol. Neurosurg. Psychiat. 13 : 191-197.

24. Ramirez, DeA. M. 1961. Hemidecortication in monkeys. Comparison of rate and degree of recovery of neurological deficit as related to age at time of operation. Excerpt. Med. Int. Congr. Ser., No. 38 : 150-151.

25. Riley, D. A., and M. R. Rosenzweig. 1957. Echolocation in rats. J. Comp. Physiol. Psychol. 50 : 323-328.

26. Tucker, T. J., and A. KLING. 1967. Differential effects of early and late lesions of frontal granular cortex in the monkey. Brain Res. $5: 377-389$.

27. Welt, C., J. C. Aschoff, K. KamedA, and V. B. Brooks. 1967. Intracortical organization of cat's motorsensory neurons, pp. 255-293. In "Neurophysiological Basis of Normal and Abnormal Motor Activities." M. D. Yahr and D. P. Purpura [eds]. Raven Press, New York.

28. White, H. H. 1961. Cerebral hemispherectomy in the treatment of infantile hemiplegia. Confin. Neurol. $21: 1-50$.

29. White, R. L., L. H. Schreiner, R. A. Hughes, C. S. MacCarty, and J. H. GRINDLAY. 1959. Physiologic consequences of total hemispherectomy in the monkey; operative method and functional recovery. Neurology $\mathbf{9}: 149-159$.

30. Woolsey, C. N. 1952. Patterns of localization in sensory and motor areas of the cerebral cortex, pp. 193-206. In "The Biology of Mental Health and Disease." The Twenty-seventh Annual Conference of the Milbank Memorial Fund, Hoeber, New York.

31. Zimmerman, R. A., W. W. Chambers, and C. N. Liu. 1964. An experimental study of the anatomical organization of the corticobulbar system in the albino rat. J. Comp. Neurol. 123 : 301-323. 